\title{
RESEARCH
}

Open Access

\section{Obstructive lung disease and quality of life after cure of multi-drug-resistant tuberculosis in Uganda: a cross-sectional study}

Edwin Nuwagira ${ }^{1 *}$ (D), Anna Stadelman ${ }^{2,3+}$, Joseph Baruch Baluku ${ }^{4 \dagger}$, Joshua Rhein², Pauline Byakika-Kibwika ${ }^{4}$ Harriet Mayanja ${ }^{4}$ and Ken M. Kunisaki ${ }^{5,6}$

\begin{abstract}
Background: Pulmonary multi-drug-resistant tuberculosis (MDR TB) alters lung architecture and involves lengthy treatment duration, high pill burden, drug adverse effects, travel restrictions, and stigma. Literature about pulmonary function and health-related quality of life (QoL) of patients treated for MDR TB is limited. This study sought to determine the prevalence of chronic obstructive pulmonary disease (COPD) and QoL of patients who were treated for pulmonary MDR TB.
\end{abstract}

Methods: Participants who completed 18 months of pulmonary MDR TB treatment and considered cured were eligible to be evaluated in a cross-sectional study. We performed post-bronchodilator spirometry to measure forced expiratory volume in $1 \mathrm{~s}\left(\mathrm{FEV}_{1}\right)$ and forced vital capacity (FVC). COPD was defined as $\mathrm{FEV}_{1} / \mathrm{FVC}<0.7$; health-related QoL was assessed using the Medical Outcomes Survey for HIV (MOS-HIV) and St. George's Respiratory Questionnaire (SGRQ). Linear and logistic regression models were used to assess associations with COPD, health-related QoL, and other characteristics of the cohort.

Results: A total of 95 participants were enrolled. Median age of the cohort was 39 years (interquartile range (IQR), 29-45), and 55 (58\%) were HIV-positive. COPD prevalence was 23\% (22/95). Median SGRQ score was normal at 7.8 (IQR, 3.1-14.8). Median mental and physical health summary scores were significantly impaired, at 58.6 (IQR, $52.0-$ 61.5) and 52.9 (IQR, 47.8-57.9), respectively, on a scale of 0 to 100 where 100 represents excellent physical or mental health. In this sample, 19\% (18/95) of participants were in the lowest relative socioeconomic position (SEP) while 34\% (32/95) were in the highest relative SEP. Belonging in the lowest SEP group was the strongest predictor of COPD.

Conclusion: Individuals who have completed MDR TB treatment have a high prevalence of COPD and low mental and physical health summary scores. Our study highlights the need for pulmonary rehabilitation programs in patients with a low socioeconomic position (SEP) after MDR TB treatment.

Keywords: Multi-drug-resistant tuberculosis, HIV, Uganda, COPD, Quality of life

\footnotetext{
* Correspondence: enuwagira@must.ac.ug

${ }^{\dagger}$ Edwin Nuwagira, Anna Stadelman and Joseph Baruch Baluku contributed equally to this work.

'Department of Medicine, Mbarara University of Science and Technology,

P.O Box 1410, Mbarara, Uganda

Full list of author information is available at the end of the article
}

(c) The Author(s). 2020 Open Access This article is licensed under a Creative Commons Attribution 4.0 International License, which permits use, sharing, adaptation, distribution and reproduction in any medium or format, as long as you give appropriate credit to the original author(s) and the source, provide a link to the Creative Commons licence, and indicate if changes were made. The images or other third party material in this article are included in the article's Creative Commons licence, unless indicated otherwise in a credit line to the material. If material is not included in the article's Creative Commons licence and your intended use is not permitted by statutory regulation or exceeds the permitted use, you will need to obtain permission directly from the copyright holder. To view a copy of this licence, visit http://creativecommons.org/licenses/by/4.0/. 


\section{Background}

The global prevalence of pulmonary multi-drug-resistant tuberculosis (MDR TB) continues to rise and is of major global concern, with approximately half a million people infected worldwide with MDR/rifampicin resistant (RR) tuberculosis in 2018 alone [1]. MDR TB disease destroys the lung architecture, characterized by bronchiectasis, pleural disease, and reduced lung volume $[2,3]$. It is therefore not surprising that as high as $98 \%$ of patients completing MDR TB treatment have impaired lung function [2]. Impaired lung function can be obstructive or restrictive. Our study was only focused on obstructive function. In Uganda, the prevalence of COPD among adults aged $30-39$ years is approximately $16 \%$, with biomass and tobacco use being the major risk factors [4]. With this known, the association between chronic obstructive pulmonary disease (COPD) and MDR TB has not been elucidated.

The standard treatment of MDR TB is lengthy with a significant pill burden and long hospitalization. MDR TB patients report depression, stigma, discrimination, side effects of the drugs, inability to work, social isolation, and financial constraints [5-7]. These factors most likely affect health-related quality of life (QoL) of individuals that have completed MDR TB treatment.

The World Health Organization's (WHO) End TB Strategy focuses on reducing TB deaths, incident TB cases, and household catastrophic costs due to TB [1]. However, none of these strategies considers long-term end-organ complications of MDR TB such as COPD or the psychosocial impact of the disease. Currently, there is a paucity of recommendations for assessing QoL or lung function after MDR TB treatment, and studies are needed to inform the formulation of guidelines to address this [8]. We aimed to determine the prevalence of COPD and assess the quality of life of patients after completing pulmonary MDR TB treatment at two large teaching hospitals in Uganda.

\section{Methods}

\section{Study aim}

The aim of the study was to determine the prevalence of COPD and assess the QoL of patients after completion of MDR TB treatment in Uganda.

\section{Study population}

Between January 2018 and April 2018, we used a crosssectional study design to enroll 95 participants that had completed MDR TB treatment at Mulago National Referral Hospital or Mbarara Regional Referral Hospital in Uganda. Potential participants were identified from the MDR TB registers at each study site and consecutively called. Those who were $\geq 18$ years of age, completed MDR TB treatment, and declared cured were contacted using the mobile telephone number listed in the registry and screened for eligibility. Potential participants were screened for the following exclusion criteria: extrapulmonary tuberculosis and known contraindications to post-bronchodilator spirometry testing such as documented active tuberculosis, pneumothorax, known allergy to Albuterol, known cardiac pathology, and ocular symptoms suggestive of retinal detachment. We consecutively called the 138 potential participants of whom 95 reported to the study sites. All 95 participants were consented and considered for the study.

\section{Study setting}

We conducted the study at two tertiary teaching hospitals in Uganda: Mulago National Referral Hospital and Mbarara Regional Referral Hospital. Mulago National Referral Hospital's TB treatment unit is located in the capital city of Kampala, Uganda. Mbarara Regional Referral Hospital is a rural hospital located in the western region of Uganda, $265 \mathrm{~km}$ from the capital. These two have the highest patient enrollment of 18 MDR TB treatment sites in the country.

\section{Study measurements \\ Pulmonary function}

At both sites, we measured spirometry using standardized equipment (Vitalograph ${ }^{\circ}$ Pneumotrac, Model 6800, Ennis, Ireland), which met equipment and procedure requirements of the American Thoracic Society/European Respiratory Society (ATS/ERS) spirometry standards [9]. A certified respiratory physiologist performed prebronchodilator spirometry on all participants and a postbronchodilator spirometry on all those meeting criteria for obstruction by the American Thoracic guidelines. The spirometers had been factory calibrated before use in our study, and the technicians checked daily calibration with a 3-L syringe. We were interested in the lung function (forced expiratory volume in $1 \mathrm{~s}\left(\mathrm{FEV}_{1}\right)$ ) and the forced vital capacity (FVC). We included two acceptable trials if the best values were within $200 \mathrm{~mL}$ of one another and participants who had a ratio of $\mathrm{FEV}_{1}$ to $\mathrm{FVC}$ less than 0.7 were subjected to a post-bronchodilator test using four puffs $(360 \mu \mathrm{g})$ of Albuterol (Ventolin, GSK, Philadelphia PA). Spirometry was then repeated after $10 \mathrm{~min}$. COPD was defined as a ratio of $\mathrm{FEV}_{1}$ to $\mathrm{FVC}<0.7[10]$.

\section{Surveys of health-related QoL and socioeconomic position}

The Saint George's Respiratory Questionnaire (SGRQ), Medical Outcomes Survey for HIV (MOS-HIV), and socioeconomic position (SEP) survey were translated into the two different local languages spoken at the two research sites. Study staff from both sites were trained on how to administer the surveys to participants through a face-to-face interview before spirometry. 
The SGRQ measures respiratory health status. It has 50 items with 76 weighted responses and is scored from 0 to 100 , where the higher score indicates worse respiratory health status [11]. The MOS-HIV is a comprehensive assessment of HIV/AIDS-specific QoL. The MOSHIV has 30 items and is scored from $0-100$ and provides both physical health summary (PHS) and mental health summary (MHS) scores, where the higher score indicates better health status. We used a culturally adapted MOS-HIV that has been previously validated among patients with TB in Uganda $[12,13]$. SEP survey is a relative wealth index for Uganda available from www.equitytool.org/uganda/. The relative wealth index uses possession of household items to measure the relative wealth of those in the sample. Those with the highest relative wealth were placed in the fifth position, while those with the lowest relative wealth were placed in the first position. Those in the first SEP were considered living in poverty. The SEP questionnaire was modified to capture data on employment status, body mass index (as measured by study assistant), time since MDR TB treatment completion, education level, and smoking status.

\section{Statistical analysis}

Due to the exploratory nature of this study, the sample size was not calculated. Data were entered and stored in a web-based data analysis application, REDCap (Vanderbilt University, Nashville, NT, USA). Statistical analyses were carried out in SAS (Cary, NC, USA) version 9.4. Descriptive statistics of the participants, as well as the results of the lung function testing and QoL assessments, are reported as medians with interquartile ranges (IQR). Statistical significance tests included $t$ tests, nonparametric tests, or chi-square tests as appropriate. The Global Lung Function Initiative 2012 equations were used as reference values for calculating percent predicted and less than lower limit of normal (LLN) values for this cohort [14]. We used bivariate logistic regression models to assess the association of age, sex, time since MDR TB treatment completion (months), any history of smoking, HIV status, MHS scores, PHS scores, SGRQ score, and poverty status (defined as those in the first SEP) with COPD. If a $p$ value was 0.25 or smaller, the variable was included in the multivariate logistic regression model.

\section{Results}

A total of 240 patients identified on the MDR TB registers were alive after treatment. Of these, 27 had been assigned "completed treatment" as a treatment outcome and 213 were declared cured between January 2011 and December 2017. Of these potential participants, 138 had active telephone numbers. We consecutively called the 138 potential participants of whom 95 reported to the study sites. Upon physical exam, all 95 participants were eligible for the study.

The baseline characteristics of the participants are described in Table 1. Median age was 39 (IQR 29-45) years, 55 (58\%) were HIV-positive, and 57 (60\%) were male. There were no notable characteristic differences between the HIV-positive and HIV-negative groups (Table 1).

\section{Lung function and COPD prevalence}

Median $\mathrm{FEV}_{1}$ was $2.1 \mathrm{~L}$ (IQR $1.7-2.6$ ) and $74.9 \%$ of predicted normal (IQR 62.4-88.9). Median $\mathrm{FEV}_{1} / \mathrm{FVC}$ ratio was 0.8 (IQR 0.7-0.8), and the prevalence of COPD was $23 \%$ (22/95) (Table 2). In a multivariable model, COPD was associated with poverty $(\mathrm{OR}=3.1295 \% \mathrm{CI}(0.87$, 11.7); $p=0.08$ ) but not HIV status, time since treatment completion, or smoking history (Table 3). A post hoc analysis using an alternate COPD definition of $\mathrm{FEV}_{1} /$ FVC $<$ LLN is shown in Additional file 1.

\section{Health-related QoL and socioeconomic position}

The median SGRQ score was 7.8 (IQR 3.1-14.8) [15]. The median MHS score was 52.9 (IQR 47.8-57.9). Overall, $19 \%(18 / 95)$ of participants were in the first (poorest) relative socioeconomic position (SEP) while 34\% (32/95) were in the highest relative SEP (Table 4).

Bivariate analyses indicated that age, sex, HIV status, time since treatment completion (months), and poverty status were statistically significant predictors of COPD and were included in the multivariate logistic regression model, as shown in Table 3.

\section{Discussion}

In this study of patients who completed treatment for pulmonary MDR TB at two teaching hospitals in Uganda, the prevalence of COPD was high, and patients reported poor mental and physical health. To our knowledge, this is the first study to evaluate lung function and QoL among patients completing MDR TB treatment in Uganda. Our results indicate that successful microbial cure of pulmonary MDR TB, while important, does not fully address the overall health impact of MDR TB. Pulmonary rehabilitation which has been shown to improve COPD symptoms in low resource settings like ours would be beneficial for patients completing MDR TB treatment [16].

The prevalence of COPD of $23 \%$ in our study is higher than the $16.2 \%$ prevalence reported in the general population of Uganda [4]. This is due to the consequences of MDR TB disease, which causes airflow obstruction and other lung function abnormalities $[4,17,18]$. It is also higher than the $16.7 \%$ prevalence of COPD reported by Byrne et al., among patients that had completed MDR TB treatment in Peru [19]. This difference is likely attributable to the fact that participants in their study were 
Table 1 Baseline characteristics of study population

\begin{tabular}{ll}
\hline & Median (IQR) or N (\%) \\
\hline Age, years & $39(29,45)$ \\
Male & $57(60 \%)$ \\
Education & \\
Partial or complete tertiary & $14(15 \%)$ \\
Partial or complete secondary & $24(25 \%)$ \\
Partial or complete primary & $48(51 \%)$ \\
No formal education & $9(9 \%)$ \\
Employed & $57(60 \%)$ \\
Months since completion of MDR TB treatment & $20(9,28)$ \\
BMI, kg/m ${ }^{2}$ & $20.2(18.3,22.8)$ \\
Smoking status & \\
Current & $1(1 \%)$ \\
Former & $23(24 \%)$ \\
Never & $71(75 \%)$ \\
HIV-positive & $55(58 \%)$ \\
MOS-HIV &
\end{tabular}

MOS-HIV Medical Outcomes Survey HIV, SGRQ St. George's Respiratory Questionnaire, SEP socioeconomic position

at least 3 years post-treatment. In contrast, Godoy et al., in Brazil, found the prevalence of COPD $\left(\mathrm{FEV}_{1} / \mathrm{FVC}<\right.$ $70 \%$ ) to be $39 \%$ among patients cured of MDR TB, which is higher than our study finding [20]. It is important to highlight that their study population constituted of only 18 participants who had a positive history of TB treatment before the MDR TB diagnosis and of whom $67 \%$ were former smokers (compared to $24 \%$ in our study). Although exposure to biomass fuels is a more prevalent risk factor for obstructive airway disease than cigarette smoking, the association between COPD and biomass fuel exposure is not well established in Uganda $[4,21]$. A further evaluation of the primary etiologies of
Table 2 Spirometry results and COPD ( $n=95)$

\begin{tabular}{|c|c|}
\hline Measure of lung function & Median (IQR) or N (\%) \\
\hline $\mathrm{FEV}_{1}, \mathrm{~L}$ & $2.1(1.7,2.6)$ \\
\hline $\mathrm{FEV}_{1} \geq 80 \%$ of predicted & $36(38 \%)$ \\
\hline FEV $150-79 \%$ of predicted & $47(49 \%)$ \\
\hline $\mathrm{FEV}_{1}, 30-49 \%$ of predicted & $12(13 \%)$ \\
\hline $\mathrm{FEV}_{1}<30 \%$ of predicted & $0(0 \%)$ \\
\hline $\mathrm{FEV}_{1}$ percent predicted & $74.9(62.4,88.9)$ \\
\hline FVC, L & $2.9(2.4,3.3)$ \\
\hline FVC percent predicted & $81.9(69.0,89.8)$ \\
\hline $\mathrm{FEV}_{1} / \mathrm{FVC}$ ratio & $0.78(0.70,0.83)$ \\
\hline $\mathrm{FEV}_{1} / \mathrm{FVC}$ ratio percent predicted & $93.5(84.1,101.7)$ \\
\hline $\mathrm{FEV}_{1} / \mathrm{FVC}<\mathrm{LLN}$ & $29(31 \%)$ \\
\hline $\mathrm{FEV}_{1} / \mathrm{FVC}<0.70(\mathrm{COPD})$ & $22(23 \%)$ \\
\hline $\mathrm{FEV}_{1} \geq 80 \%$ of predicted & $0(0 \%)$ \\
\hline $\mathrm{FEV}_{1} 50-79 \%$ of predicted & $12(55 \%)$ \\
\hline $\mathrm{FEV}_{1}, 30-49 \%$ of predicted & $10(45 \%)$ \\
\hline $\mathrm{FEV}_{1}<30 \%$ of predicted & $0(0 \%)$ \\
\hline
\end{tabular}

COPD chronic obstructive pulmonary disease, LLN lower limit of normal

obstructive airway disease among previously treated MDR TB patients is warranted, especially given the increase in pollution and poor air quality in the urban areas of Uganda. Singla et al. found that $97.6 \%$ of patients who completed MDR TB treatment in India had abnormal pulmonary function tests, but they considered a higher cutoff of $\mathrm{FEV}_{1} / \mathrm{FVC}$ ratio of $<0.8$, with a $\mathrm{FVC}$ $80 \%$ of the predicted value [2]. Additionally, they had a significantly smaller sample size of only 46 patients [2].

A low QoL in the mental and physical health domains among patients with MDR TB before, during, and after treatment has been demonstrated elsewhere in Pakistan and Namibia [22, 23]. Stigma, financial impoverishment, previous history of tuberculosis, and prolonged treatment before MDR TB diagnosis may be larger contributors to the low QoL [23-25].

Table 3 Multivariate logistic regression parameter estimates for COPD

\begin{tabular}{lll}
\hline Outcome & Odds ratios $(95 \%$ confidence interval) & $p$ value \\
\hline COPD & & \\
Age, per 5 years & $1.24(0.92,1.67)$ & 0.15 \\
Sex, male & $0.45(0.13,1.85)$ & 0.30 \\
HIV-positive & $0.52(0.17,1.62)$ & 0.26 \\
Ever smoked $^{1}$ & $1.16(0.29,4.62)$ & 0.83 \\
Poverty $^{2}$ & $3.12(0.87,11.7)$ & 0.08 \\
\hline
\end{tabular}

COPD chronic obstructive pulmonary disease, $F E V_{1} / F V C<0.7$

${ }^{1}$ Current and former smokers combined

${ }^{2}$ Patients in the lowest socioeconomic position quintile vs. the patients in all other socioeconomic position quintiles 
Table 4 Summary of quality of life-MOS-HIV, SGRQ, and SEP

\begin{tabular}{ll}
\hline Quality of life measure & Median (IQR) or N (\%) \\
\hline MOS-HIV & \\
Physical health summary score & $58.6(52.0,61.5)$ \\
Mental health summary score & $52.9(47.8,57.9)$ \\
SGRQ & \\
Total score & $7.8(3.1,14.8)$ \\
Median score for those with FEV 1 & $4.9(3.1,10.5)$ \\
$\geq 80 \%$ of predicted & \\
Median score for those with FEV 1 & $9.4(3.1,19.4)$ \\
$50-79 \%$ of predicted & $14.2(9.4,16.8)$ \\
Median score for those with FEV 1 & \\
$<49 \%$ of predicted & \\
SEP & $18(19 \%)$ \\
$1-$ poorest & $10(11 \%)$ \\
2 & $15(16 \%)$ \\
3 & $20(21 \%)$ \\
4 & $32(34 \%)$ \\
$5-$ wealthiest &
\end{tabular}

MOS-HIV Medical Outcomes Survey HIV, SGRQ St. George's Respiratory Questionnaire, SEP socioeconomic position

In bivariate analyses, a history of smoking, unemployment, and low $\mathrm{FEV}_{1} / \mathrm{FVC}$ values were all found to be associated with lower mental and physical quality of life. However, due to the low sample size and subsequent lack of power, causation for poor mental and physical quality of life was unable to be determined.

MDR TB and its treatment have a catastrophic financial impact on patients [26], yet few studies have evaluated the SEP of patients after completion of treatment. van den Hof et al. found that the total costs for diagnosis and treatment of MDR TB represented 9-25 months of pre-treatment household income and up to $76 \%$ of patients reported job loss due to illness [27]. It is possible that the observation of worsening SEP over time since MDR TB treatment completion in our study is a result of the loss of income due to illness, incurred debt during treatment, and withdrawal of financial support upon completion of treatment all of which may trigger sale of household assets [28]. Participants with low QoL (MOSHIV) scores are more likely to be unwell and less able to work. Those with a low QoL are more likely to have smoked, which would explain the association between low QOL, smoking, and its effect on mental health.

In our study, participants reported minimal impairment in respiratory health status, as assessed by the widely used St. George's Respiratory Questionnaire. Byrne et al. also found that the frequency of respiratory symptoms such as wheezing, dyspnea, and phlegm were similar between controls that had never suffered TB and former MDR TB patients [19]. It has been observed that among MDR TB patients with COPD, the objective measurement of lung function does not correlate well with their subjective assessment of their symptoms [29]. This could explain the relatively normal SGRQ scores reported in our study.

Our study had some important limitations. One limitation of our study is that the cross-sectional nature of our evaluation limits the establishment of casualty. It is possible that study participants could have had COPD before acquiring MDR $\mathrm{TB}$, given that $\mathrm{COPD}$ is a risk factor for MDR TB [30, 31]. Further, the use of mobile phone contacts during the sampling procedure may have introduced non-response bias from potential participants who did not own a mobile phone. Participants with mobile phones may have had a higher SEP score than nonusers of mobile phones [32]. We did not control for other co-morbid conditions, other than HIV, yet these could affect the physical and mental health scores. Also, other common risk factors for COPD in Uganda such as exposure to biomass fuels were not adequately measured, and this can be done in a larger prospective study. The SGRQ was interviewer-administered due to language barriers, and this non-standard method of SGRQ administration could have affected the reporting of respiratory health status. The translation of the questionnaire into a local language such as Luganda would perhaps have yielded better discrimination between participants with and without COPD [33], but the Luganda validation of the SGRQ was not published until after our study was underway. Lastly, our sample size is small, so these results should be validated in larger studies.

\section{Conclusion}

After MDR TB treatment, the prevalence of COPD is high, and patients report a low health-related QoL in the domains of mental and physical health. Our study highlights the need for long-term lung care and follow-up as part of routine MDR TB treatment. We recommend a prospective study with a larger sample size to evaluate the benefit of long-term pulmonary rehabilitation among patients who complete treatment for MDR TB.

\section{Supplementary information}

Supplementary information accompanies this paper at https://doi.org/10 1186/s41182-020-00221-y.

Additional file 1: Multivariable Logistic Regression Parameter Estimates for COPD1 (Observed FEV1/FVC < LLN).

\section{Abbreviations}

MDR TB: Multi-drug-resistant tuberculosis; COPD: Chronic obstructive pulmonary disease; QOL: Quality of life; MOS-HIV: Medical Outcomes Survey for HIV; PHS: Physical health summary; MHS: Mental health summary; SEP: Socioeconomic position; SGRQ: St. George Respiratory Questionnaire; WHO: World Health Organization; LLN: Lower limit of normal; FEV expiratory volume in $1 \mathrm{~s}$; FVC: Forced vital capacity 


\section{Acknowledgements}

We acknowledge the contribution of Dr. Lubega Mathias for his input in the drafting of the study protocol and acquiring some study ethical approvals.

\section{Disclaimer}

The views expressed are those of the authors and do not reflect the views of the US Government, Department of Veterans Affairs, or any of the authors' affiliated institutions.

\section{Authors' contributions}

EN conceived the research question, edited the protocol, collected the data at Mbarara site, interpreted the data, and contributed to the manuscript. AS took lead in drafting the research protocol, analyzed the data, interpreted the data, and contributed to the manuscript. JBB edited the study protocol, collected the data at Mulago site, interpreted the data, and took lead in drafting the manuscript. JR edited the study protocol, interpreted the data, and contributed to the manuscript. PBK edited the study protocol, interpreted the data, and contributed to the manuscript. HMK edited the study protocol and data and contributed to the manuscript. KK edited the study protocol, interpreted the study results, and contributed to the manuscript. All authors read and approved the final manuscript

\section{Funding}

Uganda Research Training Collaboration, Center of Global Health and Social Responsibility, University of Minnesota

\section{Availability of data and materials}

The datasets used during the current study are available from the corresponding author on reasonable request.

\section{Ethics approval and consent to participate}

The study protocol and tool were approved by the Makerere University School of Medicine Research and Ethics Committee (SOMREC), the ethics committees of the participating hospitals, the Uganda National Council of Science and Technology (UNCST) (study number: HS122ES), and the University of Minnesota institutional review board. Study participants provided written informed consent before study measurements were taken, and all participants received transport and airtime reimbursement.

\section{Consent for publication}

All the authors have agreed to have this work published.

\section{Competing interests}

K. Kunisaki reports consultancies with GlaxoSmithKline and Nuvaira, Inc. The other authors declare no competing interests.

\section{Author details}

'Department of Medicine, Mbarara University of Science and Technology, P.O Box 1410, Mbarara, Uganda. ${ }^{2}$ Division of Infectious Diseases and International Medicine, Department of Medicine, University of Minnesota Minneapolis, MN, USA. ${ }^{3}$ Division of Epidemiology and Community Health, School of Public Health, University of Minnesota, Minneapolis, MN, USA. ${ }^{4}$ Department of Medicine, Makerere University College of Health Sciences, Kampala, Uganda. ${ }^{5}$ Section of Pulmonary, Critical Care and Sleep Medicine, Minneapolis VA Health Care System, Minneapolis, MN, USA. ${ }^{6}$ Division of Pulmonology, Allergy, Critical Care and Sleep Medicine, Department of Medicine, University of Minnesota, Minneapolis, MN, USA.

\section{Received: 27 February 2020 Accepted: 3 May 2020}

Published online: 19 May 2020

\section{References}

1. World Health Organisation. Global Tuberculosis Report 2019 [Internet]. [cited 2020 Apr 8] p. 59, Box2. Available from: https://apps.who.int/iris/bitstream/ handle/10665/329368/9789241565714-eng.pdf?ua=1.

2. Singla R, Mallick M, Mrigpuri P, Singla N, Gupta A. Sequelae of pulmonary multidrug-resistant tuberculosis at the completion of treatment. Lung India Off Organ Indian Chest Soc. 2018;35(1):4

3. Wáng YXJ, Chung MJ, Skrahin A, Rosenthal A, Gabrielian A, Tartakovsky M. Radiological signs associated with pulmonary multi-drug resistant tuberculosis: an analysis of published evidences. Quant Imaging Med Surg. 2018:8(2):161

4. van Gemert F, Kirenga B, Chavannes N, Kamya M, Luzige S, Musinguzi P, et al. Prevalence of chronic obstructive pulmonary disease and associated risk factors in Uganda (FRESH AIR Uganda): a prospective cross-sectional observational study. Lancet Glob Health. 2015;3(1):e44-51.

5. Thomas BE, Shanmugam P, Malaisamy M, Ovung S, Suresh C, Subbaraman $R$, et al. Psycho-socio-economic issues challenging multidrug resistant tuberculosis patients: a systematic review. Plos One. 2016;11(1).

6. Morris MD, Quezada L, Bhat P, Moser K, Smith J, Perez H, et al. Social, economic, and psychological impacts of MDR-TB treatment in Tijuana, Mexico: a patient's perspective. Int J Tuberc Lung Dis. 2013;17(7):954-60,

7. Oladimeji O, Ushie BA, Udoh EE, Oladimeji KE, Ige OM, Obasanya O, et al. Psychosocial wellbeing of patients with multidrug resistant tuberculosis voluntarily confined to long-term hospitalisation in Nigeria. BMJ Glob Health. 2016; (3):e000006.

8. van Kampen SC, Wanner A, Edwards M, Harries AD, Kirenga BJ, Chakaya J, et al. International research and guidelines on post-tuberculosis chronic lung disorders: a systematic scoping review. BMJ Glob Health. 2018;3(4): e000745.

9. Miller M. ATS/ERS task force: standardisation of spirometry. Eur Respir J. 2005;26:319-38

10. Singh D, Agusti A, Anzueto A, Barnes PJ, Bourbeau J, Celli BR, et al. Global strategy for the diagnosis, management, and prevention of chronic obstructive lung disease: the GOLD science committee report 2019. Eur Respir J. 2019;53(5).

11. Jones P, Quirk F, Baveystock C. The St George's respiratory questionnaire. Respir Med. 1991:85:25-31.

12. Babikako HM, Neuhauser D, Katamba A, Mupere E. Feasibility, reliability and validity of health-related quality of life questionnaire among adult pulmonary tuberculosis patients in urban Uganda: cross-sectional study. Health Qual Life Outcomes. 2010;8(1):93.

13. Mast CT, Kigozi G, Wabwire-Mangen F, Black R, Sewankambo N, Serwadda $D$, et al. Measuring quality of life among HIV-infected women using a culturally adapted questionnaire in Rakai district. Uganda. AIDS Care. 2004; 16(1):81-94.

14. Quanjer PH, Stanojevic S, Cole TJ, Baur X, Hall GL, Culver BH, et al. Multiethnic reference values for spirometry for the 3-95-yr age range: the global lung function 2012 equations. 2012.

15. Ferrer M, Villasante C, Alonso J, Sobradillo V, Gabriel R, Vilagut G, et al. Interpretation of quality of life scores from the St George's Respiratory Questionnaire. Eur Respir J. 2002;19(3):405-13.

16. Jones R, Kirenga BJ, Katagira W, Singh SJ, Pooler J, Okwera A, et al. A prepost intervention study of pulmonary rehabilitation for adults with posttuberculosis lung disease in Uganda. Int J Chron Obstruct Pulmon Dis. 2017; 12:3533.

17. Byrne AL, Marais BJ, Mitnick CD, Lecca L, Marks GB. Tuberculosis and chronic respiratory disease: a systematic review. Int J Infect Dis. 2015;32:138-46.

18. Amaral AF, Coton S, Kato B, Tan WC, Studnicka M, Janson C, et al. Tuberculosis associates with both airflow obstruction and low lung function: BOLD results. Eur Respir J. 2015;46(4):1104-12.

19. Byrne AL, Marais BJ, Mitnick CD, Garden FL, Lecca L, Contreras C, et al. Chronic airflow obstruction after successful treatment of multidrug-resistant tuberculosis. ERJ Open Res. 2017;3(3).

20. Godoy MD, Mello FC, Lopes AJ, Costa W, Guimarães FS, Pacheco AG, et al. The functional assessment of patients with pulmonary multidrug-resistant tuberculosis. Respir Care. 2012;57(11):1949-54.

21. Van Gemert F, Chavannes N, Kirenga B, Jones R, Williams S, Tsiligianni I, et al. Socio-economic factors, gender and smoking as determinants of COPD in a low-income country of sub-Saharan Africa: FRESH AIR Uganda. NPJ Prim Care Respir Med. 2016;26(1):1-6.

22. Ahmad N, Javaid A, Sulaiman SAS, Basit A, Afridi AK, Jaber AAS, et al. Effects of multidrug resistant tuberculosis treatment on patients' health related quality of life: results from a follow up study. PloS One. 2016;11(7).

23. Sagwa E, Ruswa N, Musasa JP, Mantel-Teeuwisse AK. Adverse events during treatment of drug-resistant tuberculosis: a comparison between patients with or without human immunodeficiency virus co-infection. Drug Saf. 2013:36(11):1087-96

24. Sharma R, Yadav R, Sharma M, Saini V, Koushal V. Quality of life of multi drug resistant tuberculosis patients: a study of north India. Acta Med Iran. 2014:448-53. 
25. Jaber AAS, Ibrahim B. Health-related quality of life of patients with multidrug-resistant tuberculosis in Yemen: prospective study. Health Qual Life Outcomes [Internet]. 2019;17(1):142. Available from: https://doi.org/10. 1186/s12955-019-1211-0

26. Batte C, Kirenga B, Katamba A, Baena IG. Catastrophic total costs due to tuberculosis among affected households in Uganda; prevalence, drivers and policy implications. 2019.

27. van den Hof S, Collins D, Hafidz F, Beyene D, Tursynbayeva A, Tiemersma E. The socioeconomic impact of multidrug resistant tuberculosis on patients: results from Ethiopia. Indonesia and Kazakhstan. BMC Infect Dis. 2016;16(1): 470.

28. Initiative ST. A human rights approach to TB: stop TB guidelines for social mobilization. World Health Organization; 2001. WHO/CDS/STB/2001.9.

29. Baradzina HL. Diagnosis of COPD in patients with multidrug-resistant tuberculosis; 2018

30. Zhao J, Zhang X, He X, Yang G, Zhang X, Xin W, et al. Multidrug-resistant tuberculosis in patients with chronic obstructive pulmonary disease in China. Plos One. 2015;10(8)

31. Pradipta IS, Forsman LD, Bruchfeld J, Hak E, Alffenaar J-W. Risk factors of multidrug-resistant tuberculosis: a global systematic review and metaanalysis. J Infect. 2018;77(6):469-78.

32. Blumenstock J, Eagle N. Mobile divides: gender, socioeconomic status, and mobile phone use in Rwanda. 2010. p. 1-10.

33. Morgan BW, Grigsby MR, Siddharthan T, Kalyesubula R, Wise RA, Hurst JR, et al. Validation of the Saint George's Respiratory Questionnaire in Uganda. BMJ Open Respir Res. 2018:5(1).

\section{Publisher's Note}

Springer Nature remains neutral with regard to jurisdictional claims in published maps and institutional affiliations.

Ready to submit your research? Choose BMC and benefit from:

- fast, convenient online submission

- thorough peer review by experienced researchers in your field

- rapid publication on acceptance

- support for research data, including large and complex data types

- gold Open Access which fosters wider collaboration and increased citations

- maximum visibility for your research: over $100 \mathrm{M}$ website views per year

At $\mathrm{BMC}$, research is always in progress.

Learn more biomedcentral.com/submissions 